\title{
Inductive Interference on Pipelines Buried in Multilayer Soil Due to Magnetic Fields From Nearby Faulted Power Lines
}

\author{
Georgios C. Christoforidis, Dimitris P. Labridis, Senior Member, IEEE, and Petros S. Dokopoulos, Member, IEEE
}

\begin{abstract}
The interference of power transmission lines to buried pipelines, sharing the same rights of way, has been a research subject for many years. Especially under fault conditions, large currents and voltages are induced on the pipelines, posing a threat to operating personnel, equipment, and the integrity of the pipeline. The soil structure is an important parameter that affects the level of this interference. In this study, the influence of a soil structure composed of layers with different resistivities, both horizontally and vertically, on the inductive part of this interference is investigated. The method used to determine the inductive interference comprises finite-element calculations and standard circuit analysis. The results show that good knowledge of the soil structure is necessary in order to estimate the above interference with minimum error. Therefore, it is desirable that soil resistivity measurements are made both at adequate depths and at locations far away from the rights-of-way.
\end{abstract}

Index Terms-Electromagnetic reactive interference, finite-element method (FEM), pipelines, power transmission faults.

\section{INTRODUCTION}

$\mathbf{T}$ HE case of electromagnetic interference between power transmission lines and pipelines has been a topic of major concern since the early 1960s, mainly due to the following reasons.

- The rapid increase in energy consumption, especially in western countries, led to the adoption of higher load and short-circuit current levels, thus making the problem more acute.

- The ever increasing cost of rights-of-ways, suitable for power lines and pipelines, along with recent environmental regulations, aiming to protect nature and wildlife, has forced various utilities to share close or even common corridors for both power lines and pipelines. Therefore, situations where a pipeline is laid at a close distance to a transmission line for several kilometers are frequent nowadays.

This electromagnetic interference is present both during normal operating conditions and faults, and generally it consists of an inductive, a conductive, and a capacitive component. Out of the three, the inductive part is the dominant one. The

Manuscript received July 30, 2003; revised September 13, 2004. This work was supported by the General Secretariat of Research and Technology of the Greek Ministry of Development, the European Union, and the Greek Public Power Corporation (DEI).

The authors are with the Department of Electrical and Computer Engineering, Power Systems Laboratory, Aristotle University of Thessaloniki, GR-54124 Thessaloniki, Greece (e-mail: gchristo@auth.gr).

Digital Object Identifier 10.1109/TEMC.2005.847399 capacitive component may be ignored for buried pipelines, whereas the conductive part arises only in fault conditions and, specifically, in cases where the pipeline is located near the faulted structure. The inductive interference is the result of the magnetic field generated by the power line, which induces voltages in adjacent metallic conductors, like pipelines. Under fault conditions, high voltages and currents may be induced to nearby pipelines, which may result in hazards to people or working personnel touching the pipeline or other metallic structures connected to it. If the pipeline is electrically continuous, i.e., it is not separated by insulating flanges, then the induced voltages and currents "travel" throughout its length, even if the fault occurs far away from the pipeline. In addition, there is a high risk of damaging the pipeline coating, insulating flanges, or rectifiers, whereas the corrosion of the metal is accelerated.

Over the past years, the problem was examined by researchers that produced various reports, papers, and standards. The widely known Carson's relations [1] were the basis for the initial attempts to study this interference [2]-[6]. A technical recommendation was developed in Germany based on these studies, which was revised later [7], by utilizing more advanced and sophisticated analytical models in a computer program. During the late 1970s and early 1980s, two research projects of the Electrical Power Research Institute (EPRI) and the American Gas Association (AGA) introduced practical analytical expressions that could be programmed on handheld calculators [8] and computerized techniques [9]. In the following years, EPRI and AGA joined forces and developed a computer program [10]-[12] that utilizes equivalent circuits with concentrated or distributed elements with the self and mutual inductances being calculated using classic formulas from Carson [1], Pollaczek [13], [31], and Sunde [6]. Furthermore, CIGRE's Study Committee 36 produced a report detailing the different regulations existing in several countries [14] and, some years later, published a general guide on the subject [15], with a summary of its most important parts reproduced in [16]. Moreover, a universal algorithm was proposed in [17] that may be used to simulate uniformly both the inductive and conductive interference, whereas a more general method that may be applied to pipeline networks with complex geometries was proposed in [18].

More recently, a different approach using a finite-element method (FEM) was proposed [19] as a means to provide a field solution method. However, due to the large solution area of the problem, only two-dimensional (2-D) FEM calculations were performed. This made the method applicable only to symmetrical cases (e.g., parallel routings) and to cases where 
the pipeline has a perfect coating, which is a situation that is rarely encountered in reality. Defects on pipeline coating are a common fact, especially in old pipelines, and can range from a few millimeters to several decimeters. In order to overcome the above limitations, the authors proposed a hybrid method [20], utilizing both FEM calculations and circuit theory, which was validated by comparing with other published results in [21].

The previous work mentioned before assumed that the pipeline was buried in homogeneous soils. In practice, though, ground is composed of several layers with different resistivities. The importance of modeling the soil structure accurately was shown in [22], particularly for estimating the conductive interference levels and the performance of mitigation systems. It was found that, without proper modeling of the soil structure, mitigation systems have to be conservatively estimated in order to ensure adequate protection, as conductive interference levels may vary by more than an order of magnitude. Although it is stated in [22] that soil structure has a small influence on inductive interference levels compared with the conductive part, no information is given about how small this can be. In [23], the influence of nonhomogeneous earth on the inductive interference caused to telecommunication cables by ac electric traction lines was examined. The results of the parametric analysis performed in [23] showed that the inductive interference levels are also influenced by the soil structure, though not as much as the conductive interference levels.

However, the different soil layers are not limited to horizontal layers but to vertical ones as well. Previous work was limited mainly to horizontal layers, with vertical layers being studied only briefly for grounding systems [24]. The purpose, therefore, of the present study is to examine the level of influence that soil layers with different resistivities, both horizontally and vertically, have on the inductive interference levels on a buried pipeline due to the presence of a nearby power line.

In Section II, the description of the configuration studied is described, whereas Section III presents in detail the method used for the calculation of the inductive interference and the discrete steps that it comprises. Section IV presents a detailed analysis of the soil resistivity influence, with Section IV-A containing results obtained by modeling the soil with two layers and Section IV-B dealing with the case of three-earth layers. Finally, Section IV-C investigates the effect of vertical soil layers.

\section{SYSTEM DESCRIPTION}

In order to investigate the effect of multilayer earth on the interference between a power line and a nearby pipeline, the system shown in Fig. 1, adapted from the configuration in [12], is used. An electrically continuous section of a pipeline, isolated from the rest of the pipeline with insulating junctions, runs parallel to the power transmission line and shares the same rights-of-way with a length of $l_{p}=25 \mathrm{~km}$, whereas the separation distance $d$ between them is $25 \mathrm{~m}$, as shown in Fig. 2 . The pipeline is subject to the magnetic field from the nearby power line, which results in induced currents on the pipeline and voltages appearing across pipeline's surface and remote ground. The power transmission line consists of a pair of HAWK ACSR conductors per phase bundle. The parameters of the problem

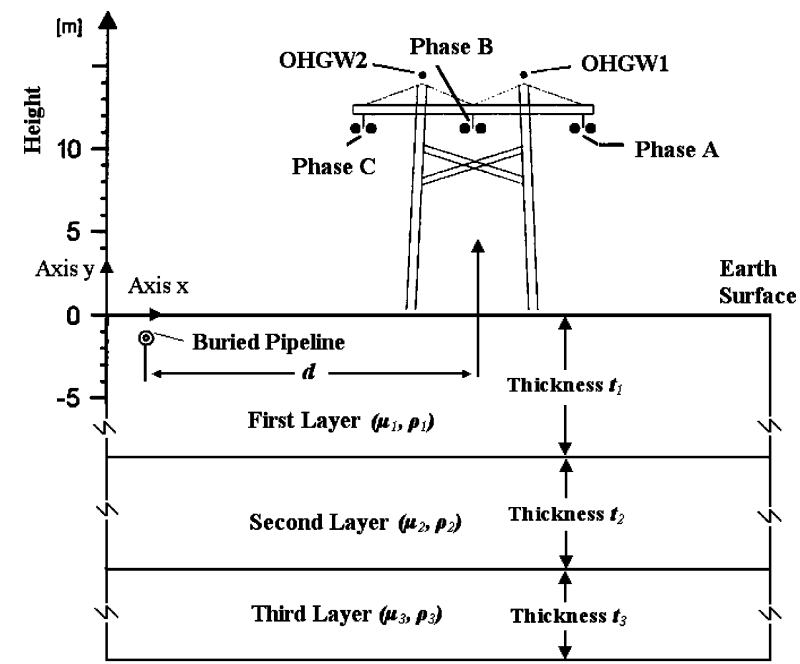

a)

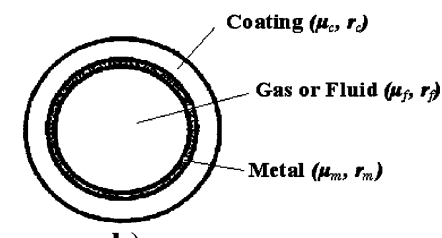

b)

Fig. 1. (a) Cross section of the system under investigation. (b) Detailed pipeline cross section.

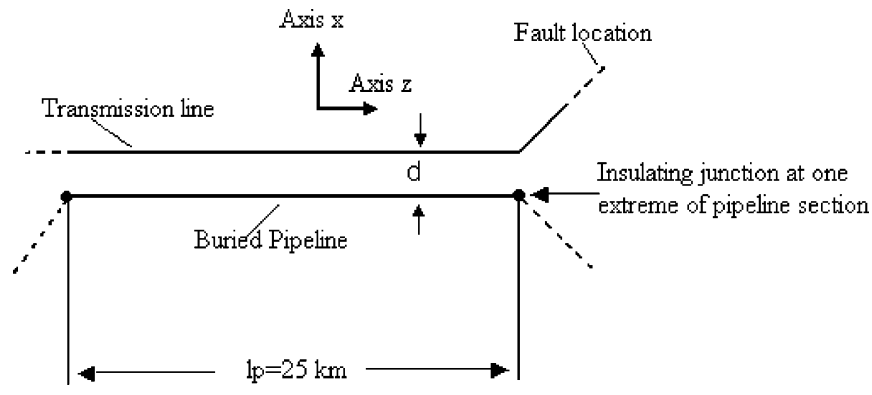

Fig. 2. Top view of the parallel exposure.

were taken from [12, Appendix] and are repeated here in the Appendix.

The soil structure, as shown in Fig. 1, consists of three horizontal layers with different electromagnetic properties, having thickness of $t_{1}, t_{2}$, and $t_{3}$, respectively. However, soil structures consisting of two horizontal layers or three vertical layers are also studied in the following sections.

A 2-D problem is considered without significant error, by neglecting end effects, consisting of infinite length conductors. This assumption is reasonable for inductive interference calculations and for the lengths of parallel exposures encountered in practical applications.

\section{DESCRIPTION OF METHOD}

The proposed method combines FEM calculations and standard circuit analysis in order to calculate the inductive coupling between a transmission line and a nearby pipeline.

The required input data for the method are:

- power line and pipeline geometrical configuration;

- physical characteristics of conductors and pipeline; 
- air and earth characteristics;

- power system terminal parameters;

- load or fault parameters.

The output data are:

- the induced voltage and current at any point on the pipeline;

- the currents flowing to earth through the leakage resistances;

- the distribution of the current returning through the ground wire(s).

\section{A. Field Equations and Finite-Element Formulation}

A system of $N$ infinitely long conductors, carrying rms currents $I_{i}, i=1,2, \ldots, N$, over imperfect earth is considered. Taking into account that the cross section of the system under investigation, shown in Fig. 1(a), lies on the $x-y$ plane, the following system of equations describes the linear 2-D electromagnetic diffusion problem for the $z$-direction components $\bar{A}_{Z}$ of the magnetic vector potential (MVP) and $\bar{J}_{Z}$ of the total current density vector [25]:

$$
\begin{aligned}
\frac{1}{\mu_{0} \mu_{r}}\left[\frac{\partial^{2} \bar{A}_{z}}{\partial x^{2}}+\frac{\partial^{2} \bar{A}_{z}}{\partial y^{2}}\right]-j \omega \sigma \bar{A}_{z}+\bar{J}_{s z} & =0 \\
-j \omega \sigma \bar{A}_{z}+\bar{J}_{s z} & =\bar{J}_{z} \\
\iint_{S_{i}} \bar{J}_{z} d S & =\bar{I}_{i}, \quad i=1,2, \ldots, N
\end{aligned}
$$

where $\sigma$ is the conductivity, $\mu_{o}$ and $\mu_{r}$ are the vacuum and relative permeabilities, respectively, $\omega$ is the angular frequency, $\bar{J}_{s z}$ is the source current density in the $z$ direction, and $\bar{I}_{i}$ is the rms value of the current flowing through conductor $i$ of cross section $S_{i}$.

It is shown in [25] that the finite-element formulation of (1) leads to a matrix equation. Using the solution of this matrix equation, the MVP values in every node of the discretization domain, as well as the $N$ unknown source current densities of the $N$ current-carrying conductors, are calculated. Therefore, for a random element $e$, the eddy-current density $\bar{J}_{e z}^{e}$ is calculated using the relation

$$
\bar{J}_{e z}^{e}(x, y)=-j \omega \sigma \bar{A}_{z}^{e}(x, y)
$$

and the total element current density $\bar{J}_{z}^{e}$, which is the sum of the conductor- $i$ source current density $\bar{J}_{s z i}$ and of the element eddy current density $\bar{J}_{e z}^{e}$ of (2a), is obtained by

$$
\bar{J}_{z}^{e}(x, y)=\bar{J}_{e z}^{e}(x, y)+\bar{J}_{s z i} \text {. }
$$

Integrating ( $2 b$ ) over a conductor cross section, the total current flowing through this conductor is obtained.

The FEM package [26], developed at the Power Systems Laboratory of the Aristotle University of Thessaloniki, has been used for the finite-element formulation of the case under investigation. A local error estimator, based on the discontinuity of the instantaneous tangential components of the magnetic field, has been chosen as in [26] for an iteratively adaptive mesh generation.

\section{B. Determination of Self and Mutual Impedances of Conductors}

There exist many methods in literature for the determination of self and mutual impedances and line parameters, from the Carson [1] and Pollaczek [13], [31] formulas to the relatively more recent ones like those of Sunde [6] and Nakagawa [27] that also consider the case of stratified earth. These methods, however, cannot deal with complex geometrical structures, apart from horizontally layered soils. A common approximation used by Sunde and various software packages (except CDEGS [28], which can deal with horizontally layered soils) is to replace a layered soil structure with an equivalent uniform soil structure. In the described method, FEM calculations are used to obtain the self and mutual impedances of any number of conductors in a region, regardless of geometrical complexity, soil structure, or terrain irregularities. Therefore, the need for utilizing approximations regarding the geometrical characteristics of a region is removed.

Generally, if there exist $N$ conductors in the configuration, the mutual complex impedance $\bar{Z}_{i j}$ between conductor $i$ and another conductor $j$ carrying a certain current $\bar{I}_{j}$, where all other conductors are forced to carry zero currents, is given by

$$
\bar{Z}_{i j}=\frac{\bar{V}_{i}}{\bar{I}_{j}}, \quad i, j=1,2, \ldots, N
$$

assuming that the per-unit length complex voltage drop $\bar{V}_{i}$ on every conductor is known for a specific current excitation.

Similarly, the self impedance of conductor $i$ may be calculated using (3), by setting $i=j$.

The procedure is summarized below [29].

- By applying a sinusoidal current excitation of arbitrary magnitude to each conductor, while applying zero current to the other conductors, the corresponding voltages are calculated.

- The self and mutual impedances of the $j$ conductor may be calculated using (3).

The above procedure is repeated $n$ times so as to calculate the impedances of $N$ conductors.

The source current density $\bar{J}_{s z i}$ for every conductor $i$ has been calculated by solving the system of (1). Therefore, (3) becomes [29]

$$
\bar{Z}_{i j}=\frac{\bar{V}_{i}}{\bar{I}_{j}}=\frac{\frac{\bar{J}_{s z i}}{\sigma_{i}}}{\bar{I}_{j}}, \quad i, j=1,2, \ldots, N
$$

Following the above procedure, effectively linking electromagnetic field variables and equivalent circuit parameters, the self and mutual impedances per unit length of the problem are computed.

With the present capabilities of personal computers, the computational time needed to calculate the self and mutual impedances using the described method has been significantly reduced. As an example, for a system with six conductors, a time of approximately $6 \mathrm{~min}$ is required, using a personal computer with a $2.4-\mathrm{GHz}$ processor and $512 \mathrm{MB}$ of memory. The authors believe the above computational time will decrease 


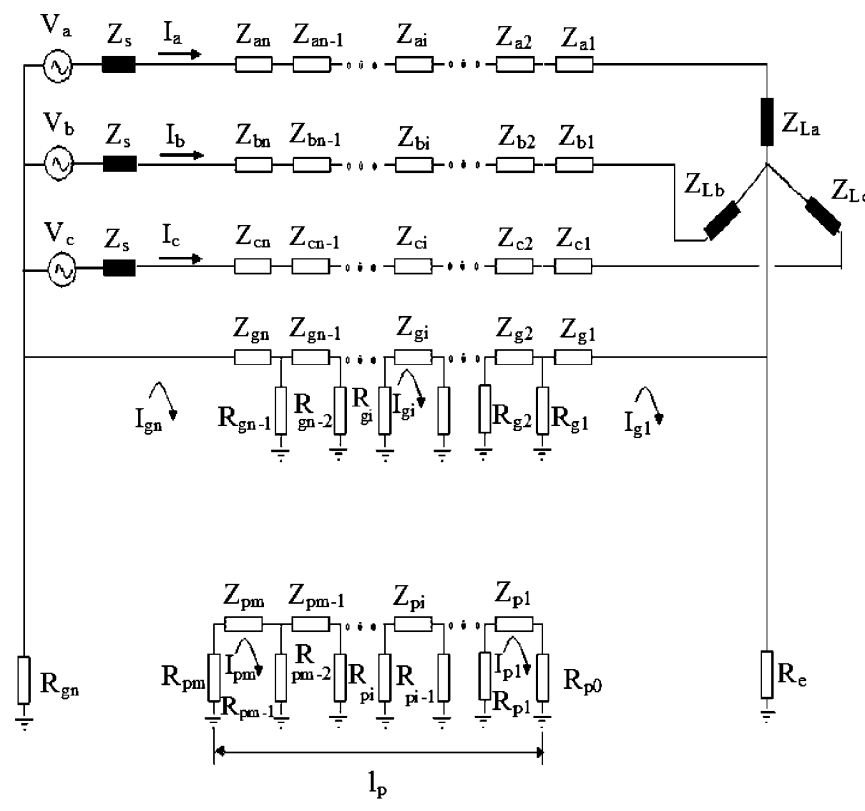

Fig. 3. Circuit representation of the problem.

rapidly in the following years, thus making the method more attractive.

\section{Circuit Representation of the Problem}

Having computed the impedances of the problem, the generalized equivalent circuit shown in Fig. 3 is constructed. The power line supplies a star connected load, with its common point earthed via a resistance $R_{e}$. However, fault situations may also be represented by setting certain values to the load impedances. For example, a single phase-to-ground fault at a certain position of phase A may be represented by setting $Z_{L A}=0$ and $Z_{L B}$ and $Z_{L C}$ to very high values, as an open circuit. In that case, $R_{e}$ will be the fault resistance. It is assumed that the towers are grounded with resistances $R_{g i}(i=0, \ldots, n)$ at frequent intervals, where $n$ is the total number of the tower groundings existing between the source and fault location.

In the circuit representation, the ground wires are replaced with an equivalent metallic return path. In order to account for the fact that the pipeline coating is not perfect, i.e., it has defects, the pipeline is modeled in sections utilizing a series of grounding-leakage resistances $R_{p i}(i=0, \ldots, m)$, where $m$ is the total number of leakage resistances and generally $m \neq n$. These resistances may also represent regular groundings used as a mitigation measure, mainly ground or polarization cells.

Generally, one may derive $n$ equations for the ground wire, $m$ for the pipeline, and three for the source loops. The unknowns of this system of equations will be the $n$ ground wire loop currents, the $m$ pipeline loop currents, and three phase currents. The method used to solve this system of equations is based on the method described in [30].

\section{INVESTIGATION OF MULTilayer SOIL STRUCTURE INFLUENCE}

The transmission-line system of Figs. 1 and 2 has been investigated for several different soil structures. Section IV-A deals with the case of a two soil layer structure, Section IV-B with a

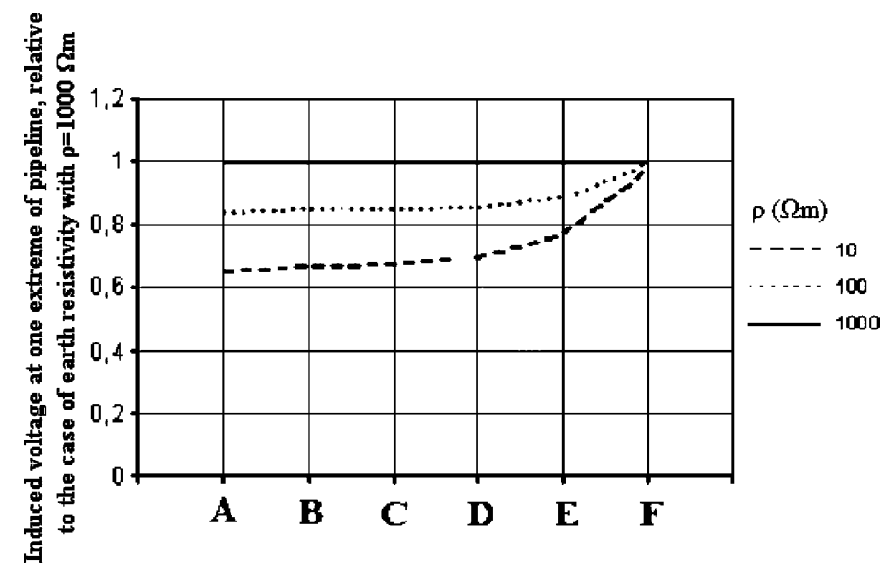

Fig. 4. Induced voltage at one extreme of the pipeline, relative to the homogeneous earth case with $\rho=10,100$, and 1000 Omega $\cdot \mathrm{m}$, for different load conditions. $A$ corresponds to the case of a fault in phase $b$ with the other two phases being unloaded, while $B$ corresponds to the case of a fault in phase $b$, with the other two phases loaded. Letters $C, D$, and $E$ correspond to the cases of unbalanced loading, with phase $b$ carrying a current with a magnitude of 2, 1.5, and 1.25 times more than the magnitude of the currents of the other two phases. Letter $F$ corresponds to the case of balanced loading.

three-soil-layer structure, and Section IV-C with the case of a soil with three vertical layers.

The effect of multilayer soil on the inductive interference studied here is demonstrated by simulating a single phase-toground fault, as is the case when the soil resistivity has the greatest influence. This may be realized by observing Fig. 4, where the induced voltage at one extreme of the pipeline is plotted for different load conditions as a function of the soil resistivity. Specifically, the points of the $x$ axis in this graph correspond to a single phase-to-ground fault with the other two phases being unloaded, a single phase-to-ground fault with the other two phases having the same load, three unbalanced load cases when the middle phase $b$ carries a current having a magnitude with a value of two, 1.5 and 1.25 times more than the magnitude of the currents of the other phases, respectively, and, finally, a balanced load case.

Consequently, in the following subsections, only the single phase-to-ground fault case with the other two phases being unloaded is considered. This is simulated, using a standard $60-\mathrm{Hz}$ frequency, at a tower that is $l=30 \mathrm{~km}$ away from the source. Higher harmonics are not considered in the analysis, as it is a subject that requires further investigation especially for transient conditions. Only the effects due to the inductive interference are taken into consideration, since the fault occurs outside the parallel exposure and, therefore, conductive interference may be neglected. Moreover, the capacitive coupling may be ignored, since the pipeline is buried. The fault impedance is modeled as pure resistance $\left(R_{e}\right)$, as it may be considered to be the grounding resistance of the faulted tower.

\section{A. Two Earth Layers}

Initially, the case of earth stratification with two horizontal layers is examined. The first layer has a resistivity ranging from 100 to $1000 \Omega \cdot \mathrm{m}$, while its thickness varies from 10 to 1000 $\mathrm{m}$. The second layer's thickness is $t_{2}=10000 \mathrm{~m}-t_{1}$, where $t_{1}$ is the thickness of the first layer, as a square with a $20-\mathrm{km}$ 


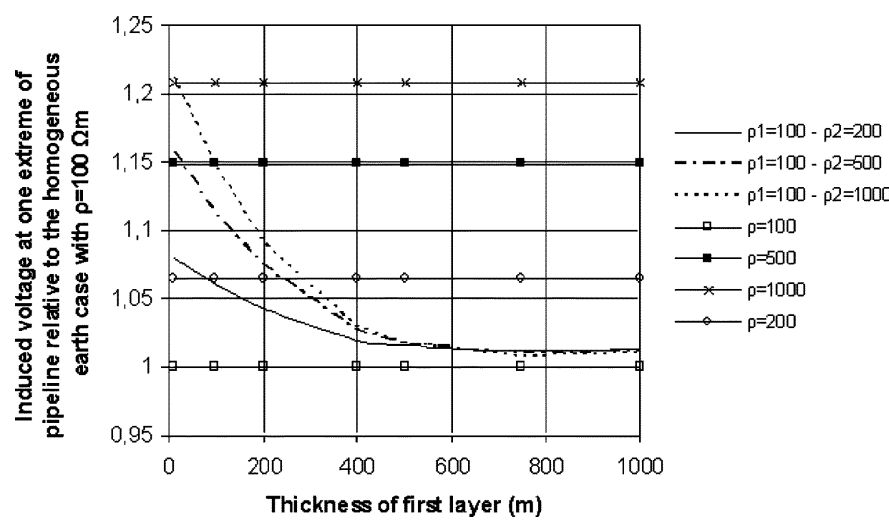

Fig. 5. Induced voltage at one extreme of pipeline, relative to the homogeneous earth case with $\rho=100 \Omega \cdot \mathrm{m}$, versus thickness of the first earth layer, as a function of the resistivity of the second earth layer. A single earth-to-ground fault is assumed in the nearby power line.



Fig. 6. Induced voltage at one extreme of pipeline, relative to the homogeneous earth case with $\rho=100 \Omega \cdot \mathrm{m}$, versus the thickness of the first earth layer, as a function of the resistivity of the second earth layers. A single earth-to-ground fault is assumed in the nearby power line.

side was used in the finite-element formulation. The most interesting results of this parametric analysis are presented in Figs. 5 and 6. The graphs in these figures depict the value of the induced voltage on one of the pipeline's extreme points versus the thickness of the first layer. This induced voltage is normalized with respect to the fault current. In the first graph, the resistivity of the first layer has a constant value of $\rho_{1}=100 \Omega \cdot \mathrm{m}$, with the resistivity of the second layer having values of $\rho_{2}=200,500$, and $1000 \Omega \cdot \mathrm{m}$. The opposite scenario is depicted in Fig. 6. In both graphs, the straight horizontal lines represent the cases of homogeneous earth having resistivities of $100,200,500$, and $1000 \Omega \cdot \mathrm{m}$, respectively.

By inspecting these two figures, one may realize that, when the first layer exceeds a 500-m thickness approximately, the inductive interference may be determined, with small error, without taking into consideration the existence of the second layer. On the other hand, when the first layer has a small thickness and there is a big difference between the resistivities of the two layers, a considerable error may be observed, as was expected. For example, when the two layers have resistivities of $\rho_{1}=100 \Omega \cdot \mathrm{m}$ and $\rho_{2}=1000 \Omega \cdot \mathrm{m}$, respectively, and the first layer has a thickness of only $10 \mathrm{~m}$, there is a deviation of around $20 \%$ from the value calculated by having a homogeneous earth of $\rho=100 \Omega \cdot \mathrm{m}$. Therefore, in order to be able

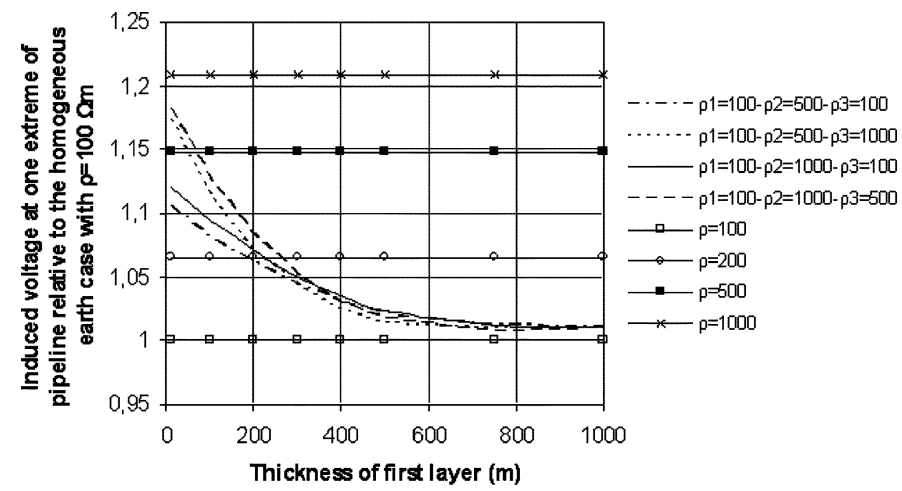

Fig. 7. Induced voltage at one extreme of pipeline relative to the homogeneous earth case with $\rho=100 \Omega \cdot \mathrm{m}$, versus thickness of the first layer, when the second layer has a thickness of $500 \mathrm{~m}$. A single earth-to-ground fault is assumed in the nearby power line.

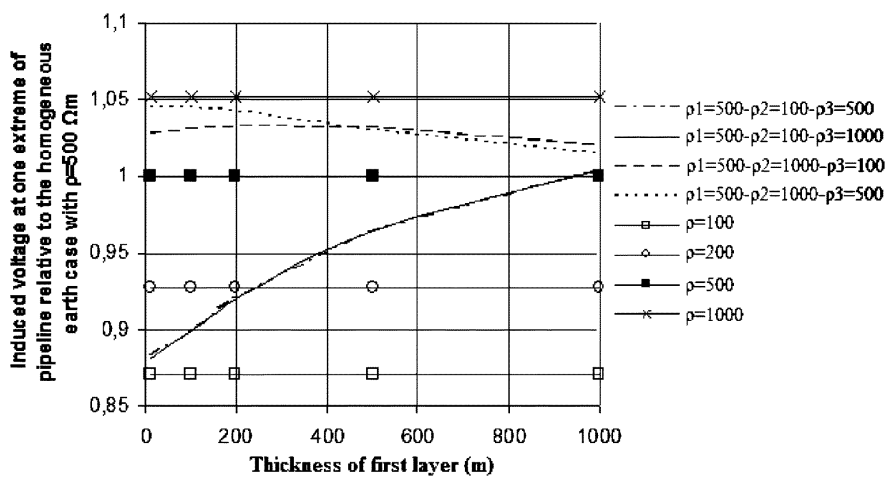

Fig. 8. Induced voltage at one extreme of pipeline relative to the homogeneous earth case with $\rho=500 \Omega \cdot \mathrm{m}$, versus thickness of the first layer, when the second layer has a thickness of $1000 \mathrm{~m}$. A single earth-to-ground fault is assumed in the nearby power line.

to calculate the inductive interference with minimum error, resistivity measurements should be made at adequate depths.

\section{B. Three Earth Layers}

In this section, some interesting graphs are presented that were obtained by modeling the earth with three horizontal layers. In order to enhance the clarity of the graphs, all lines are normalized with respect to the homogeneous earth case having the same resistivity as that of the first layer. All graphs contain the lines corresponding to the homogeneous earth cases with resistivities of 100, 200, 500, and $1000 \Omega \cdot \mathrm{m}$, respectively.

For the case shown in Fig. 7, the resistivity of the first layer is kept constant at $\rho_{1}=100 \Omega \cdot \mathrm{m}$ and the thickness of the second layer is $t_{2}=500 \mathrm{~m}$. The induced voltage at one extreme of the pipeline is plotted against the thickness of the first layer, which varies from 10 to $1000 \mathrm{~m}$. The difference between Figs. 7 and 8 is that, in the latter, the first layer has a resistivity of $500 \Omega \cdot \mathrm{m}$ and the second layer has a thickness of $1000 \mathrm{~m}$. In the third graph of this section, shown in Fig. 9, the thickness of the second layer is constant at $100 \mathrm{~m}$, while the resistivity of the first layer is now $1000 \Omega \cdot \mathrm{m}$.

By examining these graphs, the following remarks may be made.

- When the first soil layer has a thickness greater than approximately $600 \mathrm{~m}$, then the inductive interference may 


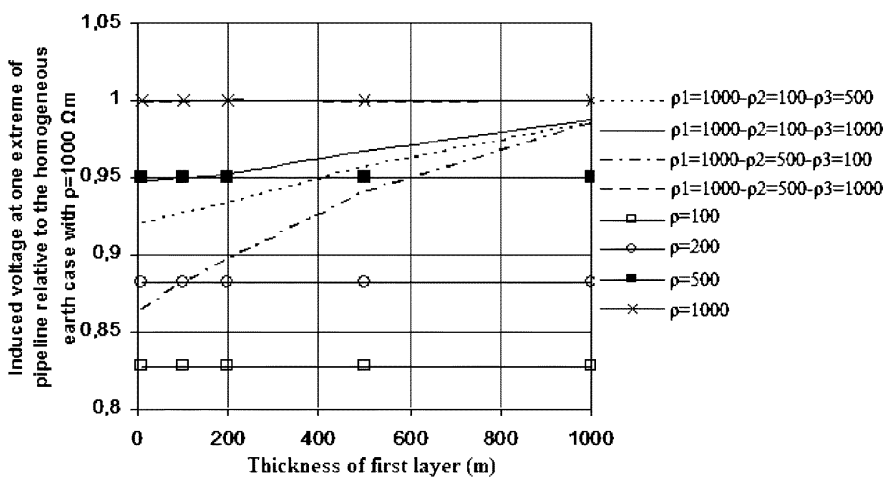

Fig. 9. Induced voltage at one extreme of pipeline relative to the homogeneous earth case with $\rho=1000 \Omega \cdot \mathrm{m}$, versus thickness of the first layer, when the second layer has a thickness of $100 \mathrm{~m}$. A single earth-to-ground fault is assumed in the nearby power line.

be determined by modeling only the first layer. The error by adopting this simplification ranges from approximately $2 \%$, when the first layer has a resistivity of $100 \Omega \mathrm{m}$, to $5 \%$, when the first layer has a resistivity of $1000 \Omega \mathrm{m}$.

- When the first soil layer has a small thickness, a deviation from the homogeneous earth case of up to approximately $18 \%$ may be observed. This variation depends on the resistivities of the other two layers. The higher the difference between the resistivities of the first and the other two layers, the greater this variation is.

\section{Vertical-Earth-Layer Analysis}

The influence of vertical soil layers on the level of inductive interference from power lines to nearby buried metallic conductors has not been covered in the past. The case of vertical layers was examined for grounding systems [24], showing that it may have a considerable effect.

In order to investigate the influence of the presence of vertical soil layers on the inductive interference between a power line and a buried pipeline, a parametric analysis was performed. The soil is divided into three vertical layers, with the pipeline being buried in the middle one. The middle layer has a resistivity ranging from 10 to $1000 \Omega \cdot \mathrm{m}$, while its width ranges from 100 to $2000 \mathrm{~m}$ and its axis of symmetry coincides with the transmission-line tower. The other two layers have at all runs the same resistivity, whereas their widths are equal and are affected directly from the width of the middle layer, as a square with a $20-\mathrm{km}$ side was used in the finite-element formulation. Therefore, when, for example, the width of the middle layer is $1000 \mathrm{~m}$, the width of the other two layers is $9500 \mathrm{~m}$.

Figs. 10-12 show the induced voltage at one extreme of the pipeline versus the width of the middle layer as a function of the resistivity value of the other two layers. The soil resistivity $\rho_{2}$ of the middle layer in Figs. 10-12 is 1000, 100, and $500 \Omega$. $\mathrm{m}$, respectively. The resistivities of the left and right layers are denoted as $\rho_{1}$ and $\rho_{3}$, respectively. The graphs are normalized with respect to the homogeneous earth case. This means that, in each graph, the line showing $100 \%$ induced voltage corresponds to a homogeneous earth case having the resistivity of the middle layer.

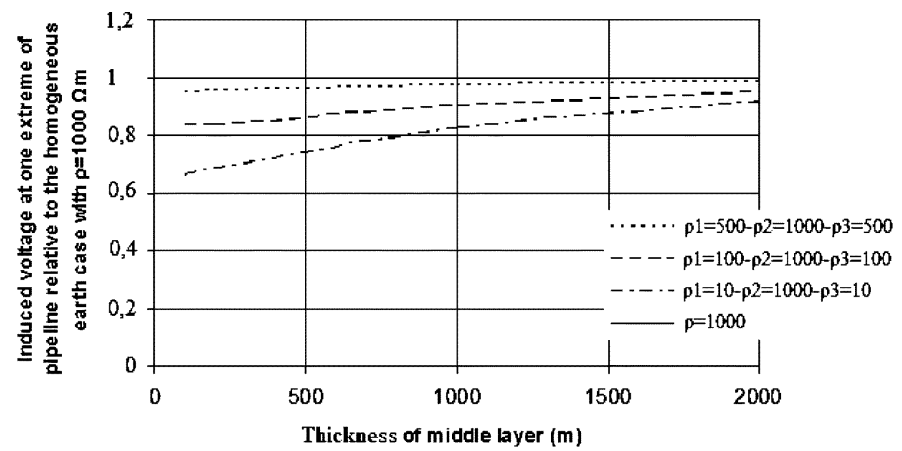

Fig. 10. Induced voltage at one extreme of pipeline, relative to the homogeneous earth case with $\rho=1000 \Omega \cdot \mathrm{m}$, versus width of the middle earth layer as a function of the resistivities of the other two layers. A single earth-to-ground fault is assumed in the nearby power line.

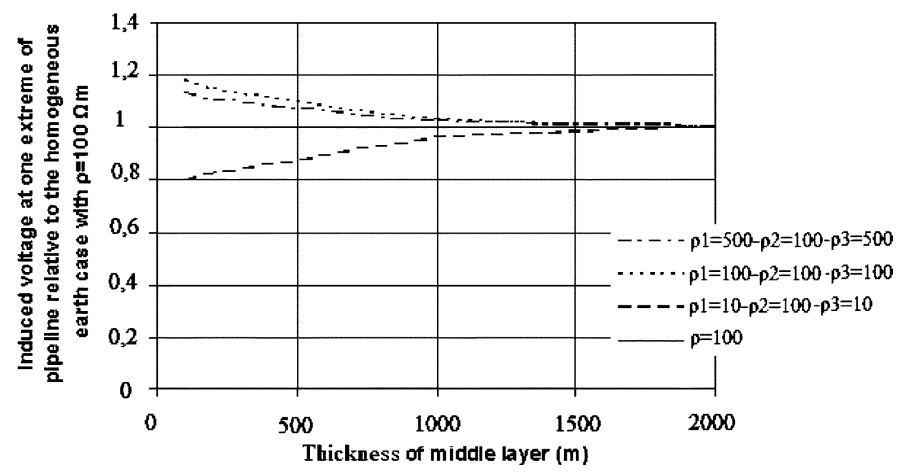

Fig. 11. Induced voltage at one extreme of pipeline, relative to the homogeneous earth case with $\rho=100 \Omega \cdot \mathrm{m}$, versus width of the middle earth layer, as a function of the resistivities of the other two layers. A single earth-to-ground fault is assumed in the nearby power line.

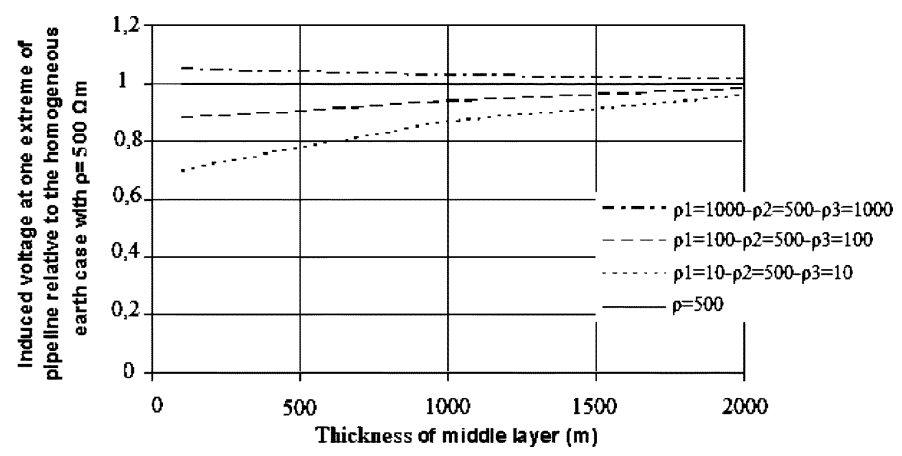

Fig. 12. Induced voltage at one extreme of pipeline, relative to the homogeneous earth case with $\rho=500 \Omega \cdot \mathrm{m}$, versus width of the middle earth layer, as a function of the resistivities of the other two layers. A single earth-to-ground fault is assumed in the nearby power line.

It may be observed from Figs. 10-12 that different vertical earth layers throughout the rights-of-way should be taken into consideration, especially for cases when the width of the middle layer is small and its resistivity varies considerably from that of the other layers. For instance, when the middle layer has a width of $10 \mathrm{~m}$ and the three vertical earth layers have a resistivity of 10,1000 , and $10 \Omega \cdot \mathrm{m}$, respectively, the amount of induced voltage calculated at one extreme of the pipeline is almost $35 \%$ lower than that of the homogeneous earth case with $1000-\Omega \cdot m$ resistivity. Therefore, in order to be able to calculate the induced parameters on a pipeline with minimum 


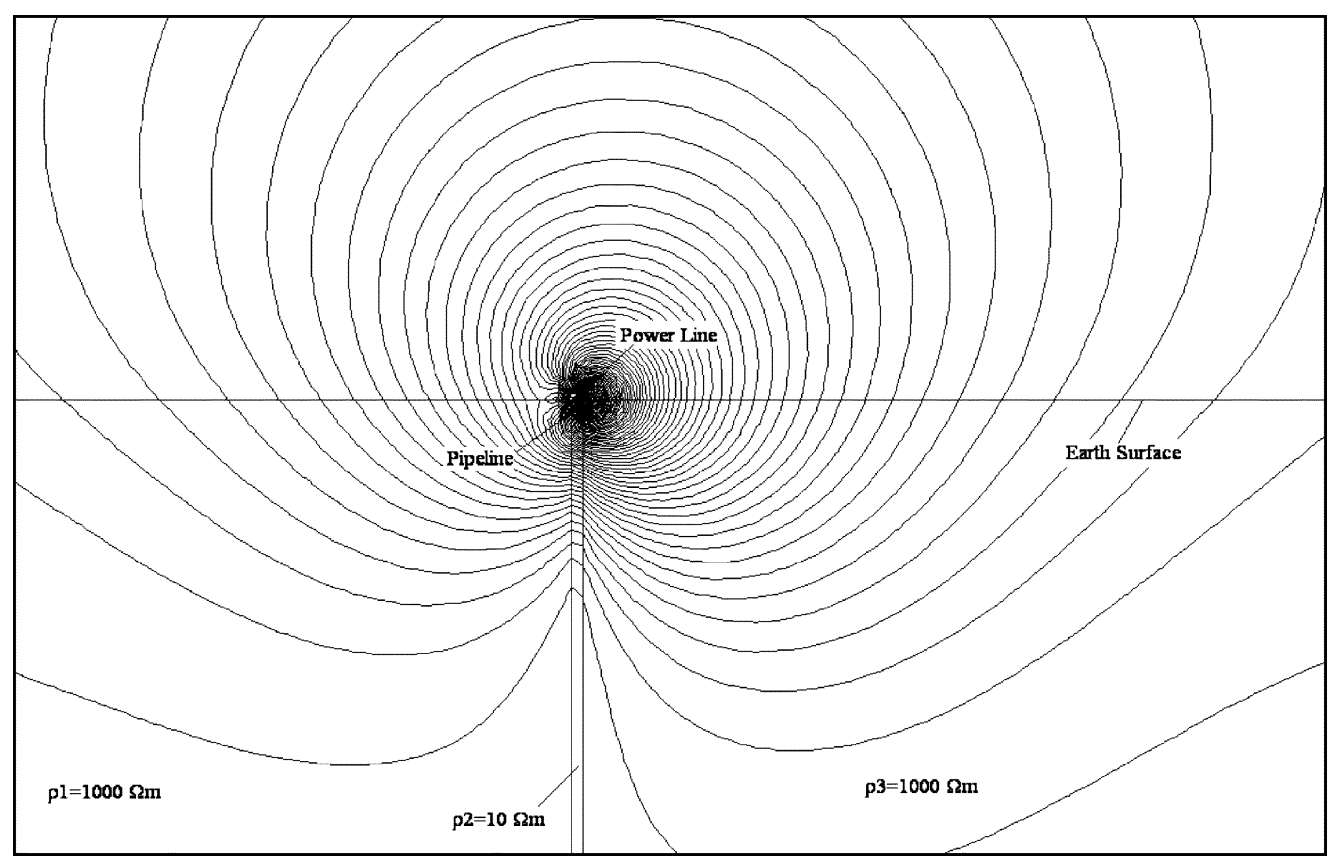

Fig. 13. Magnetic vector equipotentials $\left(A=\right.$ const $\left.-\omega t=90^{\circ}\right)$ for the case of a three-vertical-earth-layer model with resistivities $\rho_{1}=1000 \Omega \cdot \mathrm{m}$, $\rho_{2}=10 \Omega \cdot \mathrm{m}$ and $\rho_{3}=1000 \Omega \cdot \mathrm{m}$, when the middle layer has a width of $100 \mathrm{~m}$. The side of the square is $20 \mathrm{~km}$.

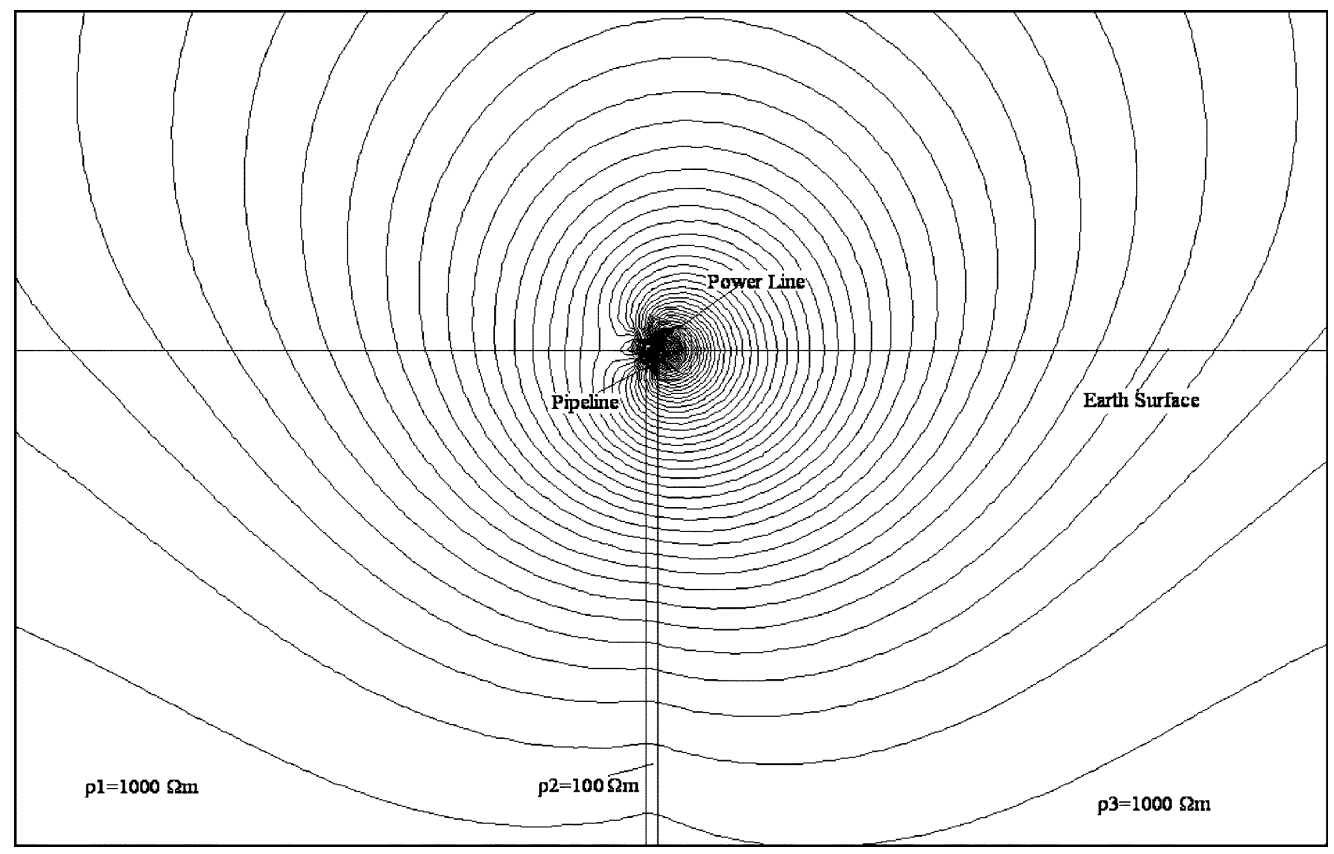

Fig. 14. Magnetic vector equipotentials $\left(A=\right.$ const $\left.-\omega t=90^{\circ}\right)$ for the case of a three vertical earth layer model with resistivities $\rho_{1}=1000 \Omega \cdot \mathrm{m}$, $\rho_{2}=100 \Omega \cdot \mathrm{m}$ and $\rho_{3}=1000 \Omega \cdot \mathrm{m}$, when the middle layer has a width of $100 \mathrm{~m}$. The side of the square is $20 \mathrm{~km}$.

error, earth resistivity measurements should be made at different locations horizontally, up to approximately $500 \mathrm{~m}$ away from the pipeline center in the $x-y$ plane. Measurements at a distance greater than $500 \mathrm{~m}$ are not of particular importance as the results are hardly influenced when the width of the middle layer exceeds that value.

Furthermore, Fig. 13 shows the flux lines for normal operating conditions (i.e., no fault) for the case where the width and the resistivity of the middle layer is $100 \mathrm{~m}$ and $10 \Omega \cdot \mathrm{m}$, respectively, and the resistivities of the other two earth layers are
$1000 \Omega \cdot \mathrm{m}$. This is a case where a strong resistive contrast exists between neighboring layers. This contrast is reflected in the electromagnetic field produced by the power line, as it may be realized by the shape of the equipotentials lines that curve toward the middle layer.

In Fig. 14, the middle layer has a resistivity of $100 \Omega \cdot \mathrm{m}$ whereas the other parameters are the same as in Fig. 13. It may be understood from this graph that, as the contrast decreases, the effect of the vertical layers also decreases, causing the flux lines to curve less sharply. 


\section{CONCLUSION}

Based on a hybrid method used for the determination of the inductive interference between a power line and a neighboring pipeline, the influence of the soil structure on inductive interference levels has been demonstrated. Cases of two and three horizontal soil layers are investigated in the paper. Resistivity measurements at adequate depths should be made if an accurate calculation of the induced parameters on the pipeline is needed. Moreover, the importance of measuring the soil resistivity at locations up to several hundred meters away from the rights-of-way has been illustrated, showing that vertical soil layers should be taken into consideration.

Situations when a multilayer soil may be replaced with an equivalent homogeneous earth soil have also been discussed concerning inductive interference predictions. These approximations may be useful for the design of effective mitigation systems, with minimum computational effort and less soil resistivity measurements.

\section{APPENDIX}

The main parameters of the case studied here are stated below. Source:
Voltage : $145220 \mathrm{~V}$
Internal Impedance :
$4+\mathrm{j} 50 \Omega$
Frequency :
$60 \mathrm{~Hz}$

Pipeline:

$\begin{array}{ll}\text { Inner Radius : } & 0.195 \mathrm{~m} \\ \text { Outer Radius : } & 0.2 \mathrm{~m} \\ \text { Coating Thickness : } & 0.1 \mathrm{~m} \\ \text { Metal Conductivity : } & 3.522 \mathrm{MS} / \mathrm{m} \\ \text { Relative Permeability } & 250 \\ \text { Coating Resistance : } & 20 \mathrm{k} \Omega \cdot \mathrm{m}^{2} \\ \text { Overhead Ground Wire }(\mathrm{s})(\mathrm{OH} G \mathrm{~W}):\end{array}$

$\begin{array}{ll}\text { Radius : } & 4 \mathrm{~mm} \\ \text { Conductivity : } & 3.522 \mathrm{MS} / \mathrm{m} \\ \text { Relative Permeability : } & 250 \\ \text { Height : } & 14 \mathrm{~m} \\ \text { No Insulation } & \end{array}$

\section{REFERENCES}

[1] J. R. Carson, "Wave propagation in overhead wires with ground return," Bell Syst. Tech. J., vol. 5, pp. 539-554, 1926.

[2] H. Böcker and D. Oeding, "Induktionsspannungen an Pipelines in Trassen von Hochspannungsleitungen," Elektrizitătswirtschaft, vol. 65, 1966.

[3] G. Kaiser, "Die elektrischen Konstanten von Rohrleitungen und ihre Messung," ETZ-A, vol. 87, pp. 792-796, 1966.

[4] J. Pohl, "Influence of high voltage overhead lines on covered pipelines," in Proc. CIGRE, Jun. 1966, Paper no. 326, pp. 1-6.

[5] B. Favez and J. C. Gougeuil, "Contribution to studies on problems resulting from the proximity of overhead lines with underground metal pipelines," in Proc. CIGRE, Jun. 1966, Paper No. 336, pp. 1-6.

[6] E. D. Sunde, Earth Conduction Effects in Transmission Systems. New York: Dover, 1968.

[7] "Arbitration Agency for Problems of Interference Between Installations of the German Federal Post Office and the Association of German Power Utilities," Verlags und Wirtschaftsgesellschaft der Elektrizitätswerke mbH-VHEW, Tech. Rec. 7, Jan. 1966.
[8] J. Dabkowski and A. Taflove, "A mutual design consideration for overhead ac transmission lines and gas pipelines,", EPRI Rep. EL-904, vol $1,1978$.

[9] M. J. Frazier, "Power line induced ac potential on natural gas pipelines for complex rights-off-way configurations,", EPRI Rep. EL-3106, Apr. 1984.

[10] F. P. Dawalibi, R. D. Southey, Y. Malric, and W. Tavcar, "Power line fault current coupling to nearby natural gas pipelines, vols. I \& II,", EPRI Rep. EL 5472, Nov. 1987.

[11] F. P. Dawalibi and R. D. Southey, "Analysis of electrical interference from power lines to gas pipelines - part I: computation methods," IEEE Trans. Power Del., vol. 4, no. 3, pp. 1840-1846, Jul. 1989.

[12] — "Analysis of electrical interference from power lines to gas pipelines-part II: parametric analysis," IEEE Trans. Power Del., vol. 5, no. 1, pp. 415-421, Jan. 1990.

[13] F. Pollaczek, "On the field produced by an infinitely long wire carrying alternating current," Elek. Nachrich.-Tech., vol. III, no. 9, pp. 339-359.

[14] P. Kouteynikoff, "Résultats d'une enquête internationale sur les règles limitant les perturbations créées sur les canalizations par les ouvrages électriques à haute tension," Proc. ELECTRA, no. 110, pp. 1-8, 1987.

[15] Guide Concerning Influence of High Voltage AC Power Systems on Metallic Pipelines, 1995.

[16] B. Jacquet and P. Kouteynikoff, "Influence of high voltage lines and installations on metal pipelines," in Proc. CIGRE Session, 1990, Paper 36-203, pp. 1-9.

[17] H. J. Haubrich, B. A. Flechner, and W. Machczyñski, "A universal model for the computation of the electromagnetic interference on earth return circuits," IEEE Trans. Power Del., vol. 9, no. 3, pp. 1593-1599, Jul. 1994.

[18] G. Lucca, L. Zucchelli, and M. Moro, "Electromagnetic interference produced on a complex metallic pipeline network by a fault in a power substation," in Proc. CIGRE Session, 2002, paper 36-101.

[19] K. J. Satsios, D. P. Labridis, and P. S. Dokopoulos, "Currents and voltages induced during earth faults in a system consisting of a transmission line and a parallel pipeline," Eur. Trans. Electrical Power (ETEP), vol. 8, no. 3, pp. 193-199, May/Jun. 1998.

[20] G. C. Christoforidis, D. P. Labridis, and P. S. Dokopoulos, "Inductive interference calculation on imperfect coated pipelines due to nearby faulted parallel transmission lines," Electric Power Syst. Res., vol. 66 , no. 2, pp. 139-148, Aug. 2003.

[21] _ "A hybrid method for calculating the inductive interference caused by faulted power lines to nearby pipelines," IEEE Trans. Power Del., vol. 20, no. 2, pp. 1465-1473, Apr. 2005.

[22] R. D. Southey, F. P. Dawalibi, and W. Vukonich, "Recent advances in the mitigation of $\mathrm{AC}$ voltages occurring in pipelines located close to electric transmission lines," IEEE Trans. Power Del., vol. 9, no. 2, pp. 1090-1097, Apr. 1994.

[23] K. J. Satsios, D. P. Labridis, and P. S. Dokopoulos, "The influence of nonhomogeneous earth on the inductive interference caused to telecommunication cables by nearby AC electric traction lines," IEEE Trans. Power Del., vol. 15, no. 3, pp. 1016-1021, Jul. 2000.

[24] F. Dawalibi and N. Barbeito, "Measurements and computations of the performance of grounding systems buried in multilayer soils," IEEE Trans. Power Del., vol. 6, no. 4, pp. 1483-1490, Oct. 1991

[25] J. Weiss and Z. Csendes, "A one-step finite element method for multiconductor skin-effect problems," IEEE Trans. Power App. Syst., vol. PAS-101, pp. 3796-3803, Oct. 1982.

[26] D. P. Labridis, "Comparative presentation of criteria used for adaptive finite element mesh generation in multiconductor eddy current problems," IEEE Trans. Magn., vol. 36, no. 1, pp. 267-280, Jan. 2000.

[27] M. Nakagawa, A. Ametani, and K. Iwamoto, "Further studies on wave propagation in overhead lines with earth return: impedance of stratified earth," Proc. Inst. Elect. Eng., vol. 120, pp. 1521-1528, 1973.

[28] F. P. Dawalibi and F. Donoso, "Integrated analysis software for grounding, EMF and EMI," IEEE Comput. Appl. Power, vol. 6, no. 2, pp. 19-24, 1993.

[29] G. K. Papagiannis, D. G. Triantafyllidis, and D. P. Labridis, "A one-step finite element formulation for the modeling of single and double circuit transmission lines," IEEE Trans. Power Syst., vol. 15, no. 1, pp. 33-38, Jan. 2000.

[30] F. P. Dawalibi and G. B. Niles, "Measurements and computations of fault current distribution on overhead transmission lines," IEEE Trans. Power App. Syst., vol. 103, no. 3, pp. 553-560, Mar. 1984.

[31] F. Pollaczek, "On the field produced by an infinitely long wire carrying alternating current," Revue Général de l'Électricité, vol. 29, no. 22, pp. 851-867, 1931. 




Georgios C. Christoforidis was born in Thessaloniki, Greece, on July 27, 1974. He received the Dipl.-Eng. degree from the Aristotle University of Thessaloniki, Thessaloniki, Greece, in 1998 and the M.S. degree in power electronics and drives from the University of Birmingham, Birmingham, U.K., in 1999. He is currently working toward the Ph.D. degree at the Aristotle University of Thessaloniki.

His research interests are in electromagnetic interference, artificial intelligence applications in power systems, and power electronics.

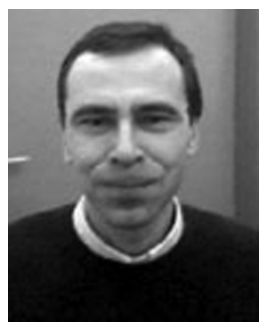

Dimitris P. Labridis (S'88-M'90-SM'00) was born in Thessaloniki, Greece, on July 26, 1958. He received the Dipl.-Eng. and Ph.D. degrees from the Aristotle University of Thessaloniki, Thessaloniki, Greece, in 1981 and 1989, respectively.

During 1982-2000, he was with the Department of Electrical and Computer Engineering, Aristotle University of Thessaloniki, first as a Research Assistant, then as a Lecturer, and later as an Assistant Professor. Since 2001, he has been an Associate Professor with the same department. His special interests are power system analysis with special emphasis on the simulation of transmission and distribution systems, electromagnetic and thermal field analysis, artificial intelligence applications in power systems, and power line communications.

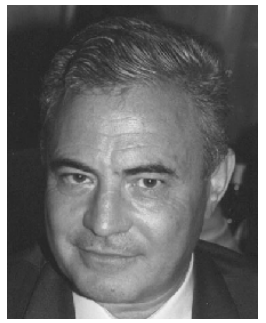

Petros S. Dokopoulos (M'94) was born in Athens, Greece, in September 1939. He received the Dipl. Eng. degree from the Technical University of Athens, Athens, Greece in 1962 and the Ph.D. degree from the University of Brunswick, Brunswick, Germany, in 1967.

From 1962 to 1967, he was with the High Voltage Laboratory, University of Brunswick. He was with the Nuclear Research Center, Julich, Germany, from 1967 to 1974 . From 1974 to 1978 , he was with the Joint European Torus. Since 1978, he has been a Professor with the Department of Electrical Engineering, Aristotle University of Thessaloniki, Thessaloniki, Greece. He has worked as consultant to Brown Boveri and Cie, Mannheim, Germany; Siemens, Erlangen, Germany; Public Power Corporation, Greece; and National Telecommunication Organization, Greece. His scientific fields of interest are dielectrics, power switches, generators, power cables, and alternative sources. He has authored 55 publications and holds seven patents in these fields. 